\title{
UTILIZATION OF SPEECH AND LANGUAGE PATHOLOGISTS (SLP) SERVICES AND FEEDING THERAPY INTERVENTIONS IN LATE PRETERM INFANTS (LPI)
}

\author{
P. Srinivasan, P. Jain, A. Melkonian, M. DiMattia \\ Pediatrics, New York Hospital Queens, Flushing, NY, USA
}

\begin{abstract}
Background: Feeding difficulties is one of the most common problems in LPIs due to low motor tone, and immature suck/swallow coordination, and is compounded by underlying respiratory illness (RI). There is wide variability in feeding ability with many having problems initiating and maintaining PO feeding. IV fluid requirement is more common.
\end{abstract}

Aims: To describe feeding problems in LPIs at initial hospitalization and evaluate utilization of SLP interventions (SLPI) to promote feeding skills.

Methods: Retrospective data were reviewed on all LPIs from 2200 NICU admissions over a period of 43 months. Data collected include\{admission characteristics, respiratory distress, sepsis, beginning and achievement of full enteral feeds (FF) and length of stay . Data on LPIs with SLPI and on matched LPI controls without interventions (NI) were analyzed.

Results: $n=399,60 \%$ of all preterm births. $12 \%$ of LPIs $(n=49)$ underwent SLPI. Mean BW/GA were 2395 gm and 35.0 wks; SLPI began by 3.5 days from the time of FF. Mean GA, BW, DR resuscitation needs, respiratory diagnoses (TTN), sepsis evaluations were similar between groups. SLPI vs NI: Time to FF 1.71 vs 1.85 days, LOS 8.8 vs 6.4 days (mean). Poor Initiation, anterior Loss of liquid, poor endurance, and easy fatigue were frequent findings. Pacing and stimulation strategies to re-initiate suck and improve placement position were common interventions. Prolonged O2 need (PO2) with a trend for early achievement of FF noted among SLPI.

Conclusions: LPIs feeding skills are burdened by presence of RI and PO2. SLP evaluations and interventions are underutilized among LPIs. 